Saudi Journal of Medicine

Abbreviated Key Title: Saudi J Med ISSN 2518-3389 (Print) |ISSN 2518-3397 (Online) Scholars Middle East Publishers, Dubai, United Arab Emirates Journal homepage: https://saudijournals.com/sjm

Original Research Article

\title{
Evaluation of Prognosis in Patients with Perforation Peritonitis Using Mannheim Peritonitis Index
}

\author{
Dr. Jacob Antony Chakiath ${ }^{1 *}$, Dr Ashok Ninan Oommen ${ }^{2}$, Dr. Harikrishnan C $\mathrm{P}^{3}$, Dr. Arun $\mathrm{P}^{4}$, Dr. Tintumole \\ C Tenny ${ }^{5}$, Dr. Greeshma Perumbilavil ${ }^{6}$ \\ ${ }^{1}$ Junior Resident, Department of Surgery, Jubilee Mission Medical College \& Research Institute, P. O, Jubilee Mission Rd, East Fort, Thrissur, Kerala \\ 680005, India \\ ${ }^{2}$ Professor, Department of Surgery, Jubilee Mission Medical College \& Research Institute, P. O, Jubilee Mission Rd, East Fort, Thrissur, Kerala \\ 680005 , India \\ ${ }^{3}$ Assistant Professor, Department of Surgery, Jubilee Mission Medical College \& Research Institute, P. O, Jubilee Mission Rd, East Fort, Thrissur, \\ Kerala 680005, India \\ ${ }^{4}$ Junior Resident, Department of Surgery, Mission Medical College \& Research Institute, Thrissur, Kerala 680005, India \\ ${ }^{5}$ Junior Resident, Department Of Pharmacology, Government Medical College, Thrissur, Kerala 680005, India \\ ${ }^{6}$ Junior Resident, Department of Pathology, Government Medical College, Thrissur, Kerala 680005, India
}

DOI: $10.36348 /$ sim.2020.v05i03.002

| Received: 16.02.2020 | Accepted: 01.03.2020 | Published: 06.03.2020

*Corresponding author: Dr. Jacob Antony Chakiath

\section{Abstract}

Background \& Objectives: Peritonitis due to hollow viscous perforation is one of the most common surgical emergencies attended by a surgeon on duty. Despite many advances in anti-microbial and supportive care, the mortality rate of diffuse peritonitis remains unacceptably high (10-20\%). The treatment of peritonitis and the evaluation of different therapeutic approaches are hampered by lack of precise classification. A scoring system should be able to assess the need, type and quality of the care required for a particular patient. Realizing the need for a simple accurate scoring system in these conditions the present study was undertaken to evaluate the prognosis in patients with perforation peritonitis using Mannheim Peritonitis Index. Materials \& Methods: Single centered, observational analytical study conducted in Jubilee Mission Medical College \& Research Institute. In our study, we have included 64 patients after meeting the inclusion criteria. As per the study protocol, patient data was collected at the time of admission and intra operatively in the Surgery Department. Mannheim Peritonitis Index score was applied to assess the outcome and data analyzed. Results and Discussion: Out of 64 patients studied, statistically significant correlation was observed between incidence of mortality and the risk factors namely, age more than 50 yrs.(25.9\%), presence of organ failure(36.4\%), preoperative duration more than 24 hrs.(18.6\%), diffuse peritonitis(18.2\%) and feacal exudates (45.5\%). No statistically significant correlation was observed between incidence of mortality and the risk factors namely female sex, non-colonic origin of sepsis, presence of malignancy. Conclusion: In our study population, all risk factors included in the Mannheim Peritonitis Index namely presence of organ failure; preoperative duration $>24 \mathrm{hrs}$; age $>50$ years, generalized extension of peritonitis and type of exudate had significant association with adverse outcome while in patients with the non-colonic origin of sepsis, presence of malignancy and of the female sex there was no significant association of these risk factors with adverse outcome (mortality). The overall mortality in our study is $12.5 \%$. Mortality in patients with MPI score < 21 was $0 \%, 21-29$ was $4.3 \%$ and $>29$ was $53.84 \%$. This reproducible scoring system allows a surgeon to determine the severity of intra-abdominal infections, to select a more aggressive management for high risk patients and to be able to inform patient's relatives with greater objectivity.

Keywords: Perforation peritonitis, Mannheim Peritonitis Index.

Copyright @ 2020: This is an open-access article distributed under the terms of the Creative Commons Attribution license which permits unrestricted use, distribution, and reproduction in any medium for non-commercial use (NonCommercial, or CC-BY-NC) provided the original author and source are credited.

\section{BACKGROUND}

Peritonitis continues to be one of the major infectious problems confronting a surgeon. Peritonitis due to hollow viscous perforation is one of the most common surgical emergencies to be attended by a surgeon on call duty. Despite many advances in antimicrobial and supportive care, the mortality rate of diffuse peritonitis remains unacceptably high (10-20\%). The treatment of peritonitis and the evaluation of different therapeutic approaches are hampered by lack of precise classification. A scoring system should be able to assess the need, type and quality of the care required for a particular patient. Several scoring systems are in place to stratify the patients with 
peritonitis due to hollow viscous perforation. Utilization of scoring systems would be of great help in salvaging a priceless life by risk stratification with preferential care and by surgical audit. Realizing the need for a simple accurate scoring system in these conditions the present study was undertaken to evaluate the prognosis in patients with perforation peritonitis using Mannheim Peritonitis Index and to validate Mannheim Peritonitis Index in our center.

\section{Mannheim Peritonitis Index}

A simplified scoring system, Mannheim's peritonitis index (MPI) was developed by Wacha and Linder [1]. It was developed based on the retrospective analysis of data from 1253 patients with peritonitis in which 20 possible risk factors were considered. Of these 20 factors, only 8 were proved to be of prognostic relevance and were entered into MPI score. These factors were classified according to their predictive power. The need for a simple accurate scoring system in the evaluation of prognosis in patients with perforation peritonitis and the need for a reproducible scoring system that allows a surgeon to determine the severity of intra-abdominal infections, to select a more aggressive surgical approach for high risk patients and to able to inform patient's relatives with greater objectivity.

\section{MATERIALS \& METHODS}

The aim was to evaluate the prognosis in patients with perforation peritonitis using Mannheim Peritonitis Index and validate Mannheim Peritonitis Index in our center. A Prospective Observational study was performed from December 2017 to May 2019 in Department of General Surgery, Jubilee Mission Medical College and Research Institution, Thrissur. Based on the sensitivity observed in a previous study "evaluation of prognosis in patient's with perforation peritonitis using mannheim's peritonitis index" with $95 \%$ confidence level and $10 \%$ relative error, the minimum sample size comes to 33 . All patients $>/=$ the age of $13 y$ rs and cases of peritonitis secondary to hollow viscous perforation attending our institute in the study period will be included in the study. All patients with primary peritonitis (Spontaneous bacterial peritonitis) and all patients with tertiary peritonitis, Patients with peritonitis due to anastomotic dehiscence or leak were excluded from the study. After approval from the Institutional Ethics Committee, all patients who all come under the inclusion criteria will be included in the study. Diagnosis will be made by a combination of history, clinical examination and on the basis of the reports of the radiological examinations after which the patients is posted for emergency surgery. Once the diagnosis of peritonitis was confirmed by the operative findings of the patients, the patients will be accepted for the study. This is a prospective observational study conducted in surgical unit in the Department of General Surgery at Jubilee Mission Medical College, Thrissur, from Dec 2017 -
May 2019. Patients enrolled in study only after informed written consent. Patients diagnosed with peritonitis secondary to hollow viscous perforation and treated surgically will be included. Initial preoperative work up and resuscitation with intravenous fluids, antibiotics, analgesics will be done in all the cases. Site of hollow viscous perforation along with extent of peritonitis and character of exudates will be documented. Appropriate surgical procedure will be performed based on etiology and patient condition. Thorough peritoneal lavage will be given in all cases. Further resuscitation and ICU care will continue as and necessary. The MPI scoring will be applied along with other clinical and biochemical parameters recorded in pre-structured Performa. Total patient MPI score will be the sum total of all the positive risk factor scores. Prediction was categorized into 3 groups: i) score $<21$ ii) score 21-29 iii) score $>29$. The various outcomes are mortality, morbidity or total recovery. The follow up will be for a period of 1 month postoperatively for morbidity. Morbidity will be assessed based on Clavien Dindo Classification. Date obtained will be analyzed for predicting the outcome. To find the outcome after categorizing the patient into 3 groups based on the MPI score. To find the cut off value of Mannheim Peritonitis Index with outcome, ROC curve will be applied.

\section{RESULTS \\ Spectrum of perforation peritonitis}

In total of 64 patients were studied the age range is from 14 years to 83 years. The mean age of the study population was 43.05 years. The highest numbers of patients were found in the age group of 31-45 years and they constitute about $28.1 \%$ of the study population. The number of patients in the age group $<50$ years were 37 i.e. $57.8 \%$ and 27 patients of the study population i.e. $42.2 \%$ were in the age group $>50$ years. In our study, out of 23 patients with gastroduodenal perforation, $16(69.56 \%)$ patients are alcoholic and $18(78.26 \%)$ patients are smokers. The increased prevalence of the perforation in the age group of 31-45 years in our study can be attributed to the fact that gastro duodenal perforations due to peptic ulcer disease is one of the major cause of perforation peritonitis and the increased prevalence of the etiological risk factors such as smoking, alcoholism abuse in this age group. Also appendicular perforation is more common in the age group of 20-30 years but no age is exempted. In our study the incidence of male sex was $64.06 \%$ while female sex was $35.6 \%$. The increased prevalence of male sex in our study is mainly due to increased number of male patients in the category of gastro-duodenal perforation.

\section{Site of Perforation}

In our study appendicular perforations account for $31.25 \%$, duodenal perforation for $18.75 \%$, gastric perforation for $17.18 \%$, colonic perforation for $15.6 \%$, ileal perforation for $9.37 \%$, jejunal perforations for $4.68 \%$, meckel's diverticulum perforation for $3.12 \%$ of 
patients. In a study by Rodolfo L et al., [2] appendicular perforations constitute $48.28 \%$ while gastric pathology and small bowel pathology constitutes $2.87 \%$ each and colonic pathology $2.30 \%$. In a study by Dani et al., [3] duodenal perforations account for $33.5 \%$, illeal perforation for $25.5 \%$, colonic perforation for $12.5 \%$, appendicular perforations for $8 \%$, gastric perforation for $6.5 \%$. The perforations of the distal gastro intestinal tract are common compared to the perforations of the proximal gastrointestinal tract which is in contrast to most of the earlier studies from India. This study is in accordance to studies from the developed countries which reveal that distal gastrointestinal tract perforations are more common.

\section{STATISTICAL VALIDATION OF MANNHIEMS PERITONITIS INDEX}

In our study, MPI had a strong correlation to mortality, statistical validation showed a sensitivity of $100 \%$ and a specificity of $87.5 \%$ at a critical score of 26 points. In our study, 15 patients had score $>26 \& 49$ patients had score $</ 26$

Table-1: Compare sensitivity, specificity with other studies

\begin{tabular}{|l|l|l|l|l|l|l|}
\hline Author & Year & Total number of patients & Sensitivity & Specificity & PPV & NPV \\
\hline Billing A et al., [15] & 1994 & 2003 & 86 & 74 & - & - \\
\hline Demmel et al., [9] & 1994 & 438 & 88 & 78 & - & - \\
\hline Corriea M et al., [16] & 2001 & 89 & 87.3 & 41.2 & - & - \\
\hline Dani et al., [3] & 2011 & 200 & 90.62 & 91.7 & 67.44 & 98.1 \\
\hline Kumar P et al., [4] & 2014 & 50 & 100 & 91 & 69 & 100 \\
\hline Present study & 2017 & 64 & 100 & 87.5 & 53.33 & 100 \\
\hline
\end{tabular}

Table-2: Clavien Dindo Classification and MPI Score

\begin{tabular}{|l|l|l|}
\hline Clavien Dindo Classification & MPI Score & No. of patients \\
\hline Grade 0 & $<21$ & 20 \\
& $21-29$ & 12 \\
& $>29$ & - \\
\hline Grade 1 & $<21$ & 2 \\
& $21-29$ & 5 \\
& $>29$ & 2 \\
\hline Grade 2 & $<21$ & 6 \\
& $21-29$ & 5 \\
& $>29$ & - \\
\hline Grade 3a & - & - \\
\hline Grade 3b & $<21$ & - \\
& $21-29$ & - \\
& $>29$ & 1 \\
\hline Grade 4a & $<21$ & - \\
& $21-29$ & - \\
& $>29$ & 3 \\
\hline Grade 4b & - & - \\
\hline Grade 5 & $<21$ & - \\
& $21-29$ & 1 \\
& $>29$ & 7 \\
\hline Total=64 & & \\
\hline
\end{tabular}

Hence our study showed that Clavien-Dindo classification is a good tool to assess the morbidity.

\section{DISCUSSION}

In our study pain in abdomen was the most common symptom and $100 \%$ of patients had pain abdomen at presentation. Distension of abdomen was present in $20.3 \%$ of patients, $44 \%$ patients had episodes of vomiting. In a study by Rajender Singh Jhobta et al., [4] pain was present in $98 \%$ of patients, followed by vomiting (59\%), abdominal distension (44\%). Perforation peritonitis is a clinical condition with a wide spectrum of presentation and high index of suspicion is always warranted. Diagnosis of perforation peritonitis is always clinical and immediate resuscitative measures should be initiated. Plan X ray abdomen was done in all cases. CECT abdomen was performed for patients in whom the diagnosis was in doubt and after initial adequate resuscitation exploratory surgery was done in an emergency basis. Delay in treatment can lead to the development of sepsis and multi-organ failure with concomitant increase in morbidity and mortality of patients.

\section{Distribution of Organ Failure}

In our study 11 patients' i.e. $17.2 \%$ of the study population showed evidence of organ failure at 
presentation. In our study patient with organ failure, mean duration of presentation was 4.6 days. In peritonitis a systemic inflammatory response induced by the peritoneal infection may progress to septic shock and multi-organ failure. The high rate organ failure in our study can be attributed to a delay in presentation of most cases.

\section{Preoperative Duration}

In our study 21 patients i.e. $32.8 \%$ presented within 24 hours while 43 patients i.e. $67.2 \%$ presented after 24 hours of onset of the disease.

In comparison with the other studies the distribution of preoperative duration are given in the table. In a study by Dani the cause of delayed presentation i.e. a preoperative duration of peritonitis more than 24 hours was mainly related to the illiteracy among the study population, lack of proper referral services and in some patients the delay was due to diagnostic dilemma which demands early use of more sophisticated investigations like CT scan, which is not available at the peripheral hospitals.

\section{Presence of Malignancy}

In our study 7 patients $(10.9 \%)$ had malignancy. 5 were cases of colonic malignancy with perforation and 2 were of carcinoma stomach with perforation. In a study by Rodolf L [2] patients had malignancy. In a study by M. M. Correia [5] patients with cancer were studied. Among them 8 were preoperative and all other were postoperative. Chronic use of NSAIDs in patients of malignancies exposes them to an increased risk of perforation.

\section{Origin of Sepsis}

In our study 10 patients i.e. $15.62 \%$ had colonic origin of sepsis while in the rest 54 patients $(84.3 \%)$ the origin of sepsis was non colonic. In the study by Rodolfo L $12.64 \%$ of patients had colonic origin of sepsis. In the study by Rajendra Singh Jobhta [4] $3.76 \%$ of patient's had colonic origin of sepsis.

\section{Type of Peritonitis}

In our study patients 44 i.e. $68.8 \%$ presented with a diffuse form of peritonitis while the remaining 20 i.e. $31.3 \%$ presented with localized peritonitis. In comparison with the other studies the distribution of types of peritonitis are given in the table. Diffuse peritonitis is associated with a severe inflammatory reaction and development of sepsis and multi-organ failure.

\section{Nature of Exudates}

In our study 5 patients i.e. $7.81 \%$ had clear exudates, 48 patients i.e. $75 \%$ had purulent exudates and 11 patients i.e. $17.19 \%$ had faecal exudates. In a study by Rodolfo L $69.5 \%$ has clear exudates and $21.8 \%$ had purulent exudates and $8.7 \%$ had faecal exudates. In a study by Rajender Singh Jhobta [4], 15\% had clear exudates, $71 \%$ had purulent and $13 \%$ had faecal exudates. In a study by Dani 40 patients i.e. $20 \%$ had clear exudates, 124 patients i.e. $62 \%$ had purulent exudates and 36 patients i.e. $18 \%$ had faecal exudates.

\section{Distribution of Patients according to MPI category}

$28(43.8 \%)$ patients had MPI score of less than

21. $23(35.9 \%)$ patients had MPI score between 21 to 29. $13(20.3 \%)$ patients had MPI score greater than 29 Of the present prognostic scoring system the Mannheim Peritonitis Index is one of the easiest to apply and the determination of risk is easily available during the initial operation. Retrospective data collection is possible and valid, as only standard information available from the operation report of the patient's record is required.

\section{Outcome}

Among the 64 patients studied by us, 8 patients died thus placing the mortality at $12.5 \%$. Rajender et al., [4] in their study of perforation peritonitis had a mortality of $10 \%$. Koperna T et al., [6] in their study of secondary peritonitis had an average total mortality rate of $18.5 \%$. The mortality rate in various studies on perforation peritonitis ranges between 10 to $20 \%$. Thus inspite of improvement in the medical management, availability of new broad spectrum antibiotics and vast development in the field of intensive care with easy availability of intensive care and life support measure, the mortality from perforation peritonitis remains high. Development of organ failure and sepsis are important determinants of mortality. Delay in the presentation for appropriate treatment should be addressed by means of strengthening the referral services and improving the means of transportation. Our efforts should be directed towards early identification of patients at risk for adverse outcome, so that prompt and effective management can be implemented there by improving the eventual outcome.

\section{Correlation between Age and Mortality}

In our study a total of 37 patients were less than 50 years of age. Out of 37 patients of age less than 50 years $1(2.70 \%)$ patients died while out of 27 patients with age more than 50 years $7(20.9 \%)$ patients died. In a study by Rodolfo L Braco [2] the mean age of the survivors was 32.7 years, among non-survivors mean age was 63 years. In a study by Dani, a total of 119 patients were less than 50 years of age. Out of 119 patients of age less than 50 years $7(5.9 \%)$ patients died while out of 81 patients with age more than 50 years 25 (30.9\%) patients died. Kusumoto Yoshiko et al., [7] in their study of patients operated on for intra-abdominal infection found that there was no mortality in less than 50 years age group, while mortality occurring only in patients older than 50 years. 


\section{Correlation between Sex and Mortality}

In our study total of 41 patients belonged to the male sex of which 5 died resulting in a mortality of $12.2 \%$. Among the 23 female patients, 3 died resulting in a mortality of $13.04 \%$. In correlation of sex with incidence of mortality, $\mathrm{p}$ value in this study was 0.922 which is statistically not significant \& this is in contrast to MPI as introduced by Wacha and Linder study [1]. Hence according to our study, female sex is not a risk factor for adverse outcome. In a study by MM Correia [5], the factor of female sex has not reached statistical significance between the groups, but it showed a good performance (accuracy of 69.7\%) when all MPI components were considered together.

\section{Correlation between Organ Failure and Mortality}

In our study a total of 11 patients showed evidence of organ failure. 4 patients among these 11 patients died thus resulting in a mortality rate of $36.36 \%$. In addition 4 patients out of 53 patients who had no evidence of organ failure died resulting in a mortality of $7.55 \%$. In our study, 2 patients died of multiple organ dysfunction (renal \& respiratory) and 5 patients died of septicaemia and acute kidney injury \& 1 patient died of respiratory failure. In the study by Muralidhar et al., [8] seven deaths (14\%) in the study, five patients died of multiple organ dysfunction and two patients died of cardiogenic shock. In the study by Rodolfo L et al., [1], 11(6.32 \%) patient's died and all of them presented with the variable of organ failure. Demmel A et al., [9] in their study found that the crude relative risk of death in patients with systemic sepsis was 13 times greater than those without. Severe sepsis was present in 424 patients $(62 \%)$ among the 628 decedents. The author concludes that severe sepsis complicates the course of $11 \%$ of all patients with peritonitis. These result mentioned above highlight the importance of early recognition, prevention, and treatment of organ dysfunction in an attempt to improve the short and long term outcome in patients with peritonitis.

\section{Correlation between Preoperative duration of Peritonitis and Mortality}

In our study out of the 21 patients with a preoperative duration of peritonitis of less than $24 \mathrm{hrs}$. no patient died. Out of the 43 patients who had preoperative duration of peritonitis of more than $24 \mathrm{hrs}$. 8 patients died thus resulting in a mortality rate of $18.6 \%$. In a study by Dani et al., [3] out of the 32 patients with a preoperative duration of peritonitis of less than $24 \mathrm{hrs}$. no patient died. Out of the 168 patients who have preoperative duration of peritonitis of more than 24 hrs. 32 patients died thus placing the mortality rate of $19 \%$. In the study by Rodolfo L [1], all the patients who died were having a preoperative duration of greater than 24 hours. Scapellato S et al., [10], suggests that intervention time may be considered the main determinant of mortality in patients with peritonitis, since intervention time is a modifiable prognostic factor while many other factors are not. Therefore in cases of perforation peritonitis after the initial resuscitation of the patient, immediate surgical procedure should be done as an emergency.

\section{Correlation between Malignancy and Mortality}

In our study 7 patients had malignancy. 2 out of the 7 patients expired placing the mortality rate in the presence of malignancy at $28.6 \%$. There were 5 cases of colonic malignancy and 2 cases of stomach malignancy. In our study, two patients with colonic malignancy expired. In correlation between presences of malignancy with incidence of mortality $\mathrm{p}$ value in our study was 0.173 , which is statistically not significant \& this is in contrast to MPI as introduced by Wacha and Linder [1]. In Wabwire B [11] (Stratified outcome evaluation in peritonitis) study had a similar result. Hence according to our study, malignancy is not a risk factor for adverse outcome

\section{Correlation between type of Peritonitis and Mortality}

In our study $44(68.75 \%)$ patients had diffuse peritonitis and 20 patients had localized peritonitis. There was no mortality in patients with localized peritonitis while among patients with diffuse peritonitis there were 8 deaths with a mortality of $18.8 \%$. In the study by Rodolfo L [1], generalized peritonitis corresponded to $34 \%$. The extension of the peritoneal inflammation process was related to increased mortality.

\section{Correlation between Origin of sepsis (colonic / non- colonic) and Mortality}

In our study 10 patients had colonic origin of sepsis out of which 2 patients died resulting in a mortality of $20 \%$ while in patients with non-colonic origin of sepsis the mortality rate in our study was $11.1 \%$. In correlation between origins of sepsis (colonic Vs non-colonic) with incidence of mortality $\mathrm{p}$ value in our study was 0.435 , which is statistically not significant and was in contrast with MPI as introduced by Wacha and Linder where colonic origin of sepsis was considered as a favorable factor. John Bohnen et al., [12] in their study of 176 patients found mortality of $10 \%$ in appendicitis and duodenal perforation, 50\% in peritonitis of intra-peritoneal origin other than appendix and the duodenum and $60 \%$ in postoperative peritonitis. Thus in this study the significance of the septic focus was high -lighted and it showed that colonic perforation is a higher risk while appendicular and duodenal perforations had a good recovery rate. Chao -Wen Hsu et al., [13] in their study of 141 patients with colorectal perforations found a mortality of $36.9 \%$. In a study by Dani et al., [3] patients had colonic origin of sepsis out of which 7 patients died resulting in a mortality of $28 \%$ while in non-colonic origin of sepsis the mortality rate in the study was $14.3 \%$. 


\section{Correlation between Character of Exudate and Mortality}

In our study among the 5 patients with clear exudates, no death. $3(6.2 \%)$ patients died among the 48 patients with purulent exudates. $5(45.5 \%)$ patients died among 11 patients with faecal exudates. Thus the mortality in patients with clear exudates was $0 \%$, purulent exudate was $6.2 \%$ while in faecal exudate the mortality was $45.5 \%$. In the study of Rodolfo L clear fluid had a mortality of $5.8 \%$, purulent fluid had a mortality of $6.3 \%$ and faecal fluid had a mortality of $25 \%$. In a study by Chao-Wen-Hsu, in fecal peritonitis the mortality was $57.10 \%$ while in purulent peritonitis it was $30.25 \%$. Killingback [14] study reported a mortality rate of over $70 \%$ in case of faecal peritonitis complicating diverticular disease. The mortality rate from purulent peritonitis in the same study was much less than that, between 10 to $30 \%$ depending upon coexisting factors such as age, cardio respiratory disease, steroid therapy and timing of surgical intervention.

\section{Correlation between MPI score with incidence of Mortality \\ In our study mortality rate among patients with} MPI score less than 21 is zero, MPI score between 21 and 29 is $4.35 \%$ and MPI score more than 29 is $53.85 \%$. Billing et al in their study of 2003 patients of perforation peritonitis found out a mortality rate of $2.3 \%$ in MPI score < 21, in MPI score between 21 and 29 the mortality was $22.5 \%$ \& it was $51.1 \%$ for MPI score greater than 29. In Dani et al study, there was no death in patients with MPI score less than 21, in MPI score between 21 to 29 the mortality was $4.3 \%$, while in patients with MPI score greater than 29 the mortality was $67.4 \%$.

\section{LIMITATIONS OF THE STUDY}

Small sample size, Short duration of the study and the diversity and individuality of biological response may prevent accurate prediction in quite a large proportion of the patients.

\section{CONCLUSION}

Mannheim Peritonitis index is a useful method to determine severity and predict outcome in patients with perforation peritonitis. All risk factors included in the Mannheim Peritonitis index namely, presence of organ failure; preoperative duration $>24 \mathrm{hrs}$; age $>50$ years, generalized extension of peritonitis and type of exudate has significant association with adverse outcome (mortality). In our study we found that Colonic origin of sepsis was associated with worse outcome probably due to presence of faecal exudates. Female sex is not a risk factor for adverse outcome as compared to male sex. Malignancy is not a risk factor for adverse outcome. Our study differs from MPI in that these above 3 were not significant risk factors for adverse outcome. Mortality can be further reduced by avoiding delay in presentation of the patients to hospital and early intervention. The MPI is one of the simple scoring systems in use that allows the surgeon to easily stratify severity of peritonitis and predict the outcome following initial surgery. Early evaluation of severity of illness using MPI allows us to estimate the probability of patient's survival. Reproducible scoring system that allow a surgeon to determine the severity of intraabdominal infections are essential to select a more aggressive management for high risk patients and to able to inform patient's relatives with greater objectivity. MPI cutoff points should be adjusted for each hospital on individual basis as in our study it was divided into 3 groups, <21, 21-29, >29. The overall mortality in our study is $12.5 \%$. Mortality in patients with MPI score < 21 was $0 \%, 21-29$ was $4.3 \%$ and $>29$ was $53.84 \%$. It was observed that, as MPI score increases, the grade according to Clavien-Dindo classification for mortality also increases. Hence our study showed that Clavien-Dindo classification is a good tool to assess the morbidity. Based on our study results we conclude that MPI is useful in patients with perforation peritonitis and should be considered reliable and simple reference for estimating morbidity and mortality. As our study differs from earlier studies in the impact of 3 risk factors i.e. female sex, non-colonic origin of sepsis, presence of malignancy with respect to the outcome, we advocate the need for further studies in this aspect on Mannhein Peritonitis Index.

\section{REFERENCES}

1. Linder, M. M., Wacha, H., Feldmann, U., Wesch, G., Streifensand, R. A., \& Gundlach, E. (1987). The Mannheim peritonitis index. An instrument for the intraoperative prognosis of peritonitis. Der Chirurg; Zeitschrift fur alle Gebiete der operativen Medizen, 58(2), 84-92.

2. Bracho-Riquelme, R. L., Melero-Vela, A., \& Torres-Ramírez, A. (2002). Mannheim peritonitis index validation study at the hospital general de Durango (Mexico). Cirugía y Cirujanos, 70(4), 217-225.

3. Dani, T., Ramachandra, L., Nair, R., \& Sharma, D. (2015). Evaluation of prognosis in patients' with perforation peritonitis using Mannheim's peritonitis index. International Journal of scientific and research publications, 5(5), 1-35.

4. Jhobta, R. S., Attri, A. K., Kaushik, R., Sharma, R., $\&$ Jhobta, A. (2006). Spectrum of perforation peritonitis in India-review of 504 consecutive cases. World journal of Emergency surgery, 1(1), 26.

5. Correia, M. M., \& Mannheim, P. D. M. U. (2001). Prediction of death using the mannheim peritonitis index in oncologic patients. Revista Brasileira de Cancerologia, 47(1), 63-68.

6. Koperna, T., \& Schulz, F. (2000). Relaparotomy in peritonitis: prognosis and treatment of patients with persisting intraabdominal infection. World journal of surgery, 24(1), 32-37. 
7. Kusumoto, Y., Nakagawa, M., Watanabe, A., Ishikawa, H., Sakaguchi, T., Yamada, T., ... \& Hongo, S. (2004). Study of Mannheim peritonitis index to predict outcome of patients with peritonitis. Japanese Journal of Gastroenterological Surgery, 37(1), 7-13.

8. Muralidhar, V. A., Madhu, C. P., \& Sudhir, S. (2014). Efficacy of Mannheim peritonitis index (MPI) score in patients with secondary peritonitis. Journal of clinical and diagnostic research: JCDR, 8(12), NC01-NC03.

9. Demmel, N., Maag, K., \& Osterholzer, G. (1994). The value of clinical parameters for determining the prognosis of peritonitis--validation of the Mannheim Peritonitis Index. Langenbecks Archiv fur Chirurgie, 379(3), 152-158.

10. Scapellato, S., Parrinello, V., Sciuto, G. S., Castorina, G., Buffone, A., \& Cirino, E. (2004). Valuation on prognostic factors about secondary acute peritonitis: review of 255 cases. Annali italiani di chirurgia, 75(2), 241-245.

11. Wabwire, B., \& Saidi, H. (2014). Stratified Outcome Evaluation of Peritonitis. Annals of African Surgery, 11(2):29-34.
12. Bohnen, J., Boulanger, M., Meakins, J. L., \& McLean, A. P. H. (1983). Prognosis in generalized peritonitis: relation to cause and risk factors. Archives of Surgery, 118(3), 285-290.

13. Hsu, C. W., King, T. M., Wang, J. H., \& Wang, H. (2007). Colorectal perforation: spectrum of the disease and its mortality. J Soc Colon Rectal Surg Taiwan, 81-88.

14. Killingback, M. (1983). Diverticular disease, In: Allan, R. N., Keighley, M. R. B., Eds. Inflammatory bowel diseases, Edinburgh: Churchill Livingstone, 504-511.

15. Billing, A., Fröhlich, D., \& Peritonitis Study Group. (1994). Prediction of outcome using the Mannheim peritonitis index in 2003 patients. British journal of surgery, 81(2), 209-213.

16. Rogy, M., Függer, R., Schemper, M., Koss, G., \& Schulz, F. (1990). The value of 2 distinct prognosis scores in patients with peritonitis. The Mannheim Peritonitis Index versus the Apache II score. Der Chirurg; Zeitschrift fur alle Gebiete der operativen Medizen, 61(4), 297-300. 